\title{
Safety, efficacy, and intraoperative characteristics of DisCoVisc and Healon ophthalmic viscosurgical devices for cataract surgery
}

This article was published in the following Dove Press journal:

Clinical Ophthalmology

22 September 2011

Number of times this article has been viewed

\section{Satish S Modi \\ James A Davison ${ }^{2}$ \\ Tom Walters ${ }^{3}$}

'Seeta Eye Centers, Poughkeepsie, NY, USA; ${ }^{2}$ Wolfe Clinic, Marshalltown, IA,

USA; ${ }^{3}$ Texas Eye Care, Austin, TX, USA
Correspondence: Satish S Modi Seeta Eye Centers, 23 Davis Avenue, Poughkeepsie, NY 12603, USA

Tel + I 8454541025

Fax + I 845454588 I

Email smodieyes@aol.com
Purpose: To evaluate the safety and efficacy of DisCoVisc ophthalmic viscosurgical device (OVD, Alcon Laboratories, Inc) with respect to a comparator, Healon OVD (Advanced Medical Optics, Inc).

Patients and methods: In this prospective study, patients with cataracts were randomized to an OVD, and then received phacoemulsification and injection of an intraocular lens. After each surgery, unmasked investigators completed subjective questionnaires about OVD characteristics during each stage of the procedure. Masked technicians evaluated objective safety parameters of intraocular pressure (IOP) and endothelial cell density, with 90 days of follow-up.

Results: The DisCoVisc OVD group (128 eyes) and the Healon OVD group (121 eyes) had statistically similar outcomes for IOP and for endothelial cell loss. Subjectively assessed viscosity was statistically different $(P<0.0001)$, with Healon OVD most often rated "cohesive" and DisCoVisc OVD most often rated "both dispersive and cohesive". Workspace maintenance differed between groups $(P<0.0001)$, with workspace most frequently rated "full chamber maintained" when using DisCoVisc OVD and most frequently rated "workspace maintained" when using Healon OVD. "Flat" or "shallow" workspace ratings occurred only in the Healon OVD group.

Conclusion: DisCoVisc OVD had both cohesive and dispersive properties, and was safe and effective for every stage of cataract surgery.

Keywords: cataract, endothelial cell density, viscoelastic, phacoemulsification

\section{Introduction}

Viscoelastics, or ophthalmic viscosurgical devices (OVDs), facilitate cataract surgery by maintaining the depth and shape of the anterior chamber. This provides a workspace for the surgeon and provides a viscous barrier that protects the delicate corneal endothelium from surgical instruments, from cataractous lens debris, and from the intraocular lens during insertion. ${ }^{1}$ Early OVDs were classified as either cohesive or dispersive, on the basis of objective rheological properties. ${ }^{2}$ Cohesive OVDs are useful in creating and maintaining space in the anterior chamber. ${ }^{1}$ Because cohesive OVDs tend to hold together as a mass, they are relatively easy to remove as a bolus at the end of surgery. ${ }^{1}$ In contrast to cohesive OVDs, dispersive OVDs spread out when injected into the eye, making these substances less effective for maintaining space but more effective for coating and protecting intraocular tissues. ${ }^{3}$ Irrigation/aspiration tends to pull away bits and fragments of dispersive OVDs, making these materials more difficult to remove at the end of surgery. ${ }^{1,3}$ The different properties of cohesive and dispersive viscoelastics broaden the opportunities for a surgeon's selection of an OVD for cataract surgery. 
The two OVD types - cohesive and dispersive - can be used for different stages of the surgery or for different types of eyes. For example, a cohesive OVD could be selected to expand a small pupil, but a dispersive OVD could be used to protect an eye with a compromised corneal endothelium. ${ }^{1}$ Some surgical strategies, such as the soft-shell technique, use two OVD types together in layers or serially. ${ }^{4}$ For convenience to the surgeon, manufacturers may package two OVD types together, as with the DuoVisc viscoelastic system (Alcon Laboratories, Inc, Fort Worth, TX).

The endothelium-protecting efficacy of an OVD can be evaluated in terms of postoperative measurements of endothelial cell density. Endothelial cell loss occurs during surgery and during the postoperative phase, and the loss can continue at a faster-than-normal rate for at least 10 years thereafter. ${ }^{5}$ If the normal endothelial cell density of $\sim 2400$ cells $/ \mathrm{mm}^{2}$ falls below 300-500 cells $/ \mathrm{mm}^{2}$, corneal edema can develop, and can be followed by decompensation into bullous keratopathy. ${ }^{6}$ Rheological properties indicate that a dispersive OVD, with its propensity to coat and protect intraocular tissues, might be better than a cohesive OVD for endothelial protection.

While an ideal OVD would completely coat and protect intraocular tissues during surgery, an ideal OVD also would be able to be completely removed from intraocular tissues at the conclusion of surgery. Residual OVD left in the eye can clog the trabecular meshwork, leading to a transient elevation in postoperative intraocular pressure (IOP) ${ }^{7-9}$ This ocular hypertension is sometimes treated with IOP reducing medication, either prophylactically or in response to postoperative observations of IOP spikes to $\geq 30 \mathrm{mmHg}^{10}$ or $\geq 35 \mathrm{mmHg} .{ }^{11}$ Alternatively or in addition to IOP treatment, a surgeon can attempt to avoid IOP spikes by selecting an OVD that is conducive to complete removal at the end of surgery. Rheological properties indicate that a cohesive OVD, with its propensity to be removed as a bolus, might be better than a dispersive OVD for avoiding IOP spikes.

Facilitation of surgical techniques, ability to protect endothelium, and avoidance of IOP spikes are all factors that need to be considered in selecting an OVD, but these considerations sometimes work at cross purposes; no single OVD is a clear choice. In an attempt to provide surgeons with a single OVD that was suitable for all phases of surgery, one manufacturer (Alcon) developed DisCoVisc OVD. This OVD exhibited both dispersive and cohesive properties in bench testing, and thus was given the new classification "viscous dispersive". ${ }^{2}$ The duality was intended to preclude the need for multiple OVDs during cataract surgery, while providing good endothelial protection and avoiding postoperative IOP spikes.
This manuscript presents the clinical data that were provided to the US Food and Drug Administration to support the approval of DisCoVisc OVD for ophthalmic use. A cohesive OVD, Healon (1\% hyaluronic acid, Advanced Medical Optics, Inc, Santa Ana, CA), was used as a comparator. Surgeons assessed the surgical characteristics of the OVDs at the conclusion of each surgery, and patients were evaluated for postoperative intraocular pressure and endothelial cell density. Overall, the study was designed to investigate whether DisCoVisc OVD was safe and effective for every stage of the phacoemulsification surgical procedure.

\section{Material and methods}

\section{Patient enrollment and baseline}

Each of the nine investigators, at nine clinical sites in the US, prospectively enrolled 20 to 44 patients. Each patient had only one eye enrolled in the study. At least 125 eyes per treatment group (250 in total) were targeted for enrollment, because calculations had indicated that 113 eyes per group would be required to yield a minimally detectable difference of $13 \%$ (noninferiority margin between groups) in eyes with $\mathrm{IOP} \geq 30 \mathrm{mmHg}$. Guidance from the relevant protocol from the International Organization for Standardization ${ }^{12}$ was used to generate these target enrollment numbers and to set the IOP safety limit. Calculations included the assumption that $30 \%$ of patients in each group would have IOP $\geq 30 \mathrm{mmHg}$.

Eligible patients were 18 years or older and were scheduled for removal of a cataract by phacoemulsification followed by implantation of a posterior chamber intraocular lens. Each patient's nonsurgical eye was required to be functional, as assessed by the investigator. Exclusion criteria related to endothelial cell density of the operative eye were a baseline endothelial cell density of less than 1500 cells $/ \mathrm{mm}^{2}$ or a poor quality photograph of preoperative endothelial cells. Exclusion criteria related to IOP in the operative eye were as follows: any abnormality that prevented reliable Goldmann applanation tonometry, glaucoma or other causes of compromised aqueous humor outflow, ocular hypertension $>21 \mathrm{mmHg}$, or lens pseudoexfoliation syndrome. Operative eyes could not have iris atrophy, a history of chronic or recurrent inflammatory eye disease, proliferative diabetic retinopathy, previous ocular trauma or surgery, or a congenital ocular anomaly. All patients signed consent forms in accordance with the Declaration of Helsinki. Each investigator received ethical approval from an Institutional Review Board.

Central corneal endothelial cell densities were measured with a noncontact Noncon Robo specular microscope (Konan 
Medical, Inc, Irvine, CA), such as a Noncon Robo SP-8000 with KSS software. Each study site obtained two images of the central cornea of each operative eye. Individuals were excluded from the study if their baseline endothelial cell images were of poor quality (having cell borders that were not easily distinguishable). Images were sent to a central treatment-masked reading center (Alcon Research, Ltd) that measured corneal endothelial cell densities. For each eye, the masked reading center selected the photo that showed better-quality cell borders and attempted to mark at least 100 contiguous cells on each image, in order to obtain an analysis of at least 50 cells. If the reading center was unable to obtain accurate measurements from the first selected photo, the second photo was used for the calculation.

Treatment-masked observers at each site assessed IOP via Goldmann applanation tonometry. In order to provide an accurate representation of the effect of the OVDs on the IOP, prophylactic IOP-reducing medications were not permitted before, during, or immediately after surgery. For patient safety, IOP-reducing therapies were allowed at 6 hours postoperative and/or at 24 hours postoperative, if IOP reached $\geq 30 \mathrm{mmHg}$. This design ensured that the 6 -hour IOP measurements were not influenced by IOP reducing medication.

\section{Randomization and surgeries}

Prior to distribution of OVD packages, patients were assigned to a number, which was randomized to either the DisCoVisc OVD group or the Healon OVD group. The two OVDs were provided to each surgical site in identical, sealed, plain cartons. The outside of each carton was labeled with the study protocol number, the investigator number, and the patient number. Each masked carton was opened just before the beginning of the intended surgery. Inside the cartons, Healon OVD was supplied as one syringe of $0.85 \mathrm{~mL}$, and DisCoVisc OVD was supplied as two syringes of $0.5 \mathrm{~mL}$ each. Because of these differences, the surgeons became unmasked to the identity of the OVDs during the procedure. For each patient, a disclosure envelope to reveal OVD assignment was available, but was to be opened only if a serious adverse event occurred.

During surgery, multiple procedures were not allowed (eg, no concurrent trabeculoplasty or corneal transplant), but minor relaxing keratotomy for the correction of astigmatism was permitted. Standard clear corneal incisions, capsulorhexis, and phacoemulsification were performed. All phacoemulsification machines were longitudinal (no torsional or transversal modes). Ultrasound time and surgery duration were not recorded. Only approved IOLs were used; investigational IOLs were not permitted.

At the completion of every surgery, each of the nine investigators completed a survey. Three questions addressed anterior chamber and dome maintenance (during capsulotomy, during phacoemulsification, and during IOL insertion), with four response options, as follows: flat, shallow, working space maintained, or full chamber maintained. Three further questions addressed the surgeon's perceived rheological properties of the viscoelastic during capsulorhexis, during phacoemulsification, and during removal of the OVD. The five response options were as follows: dispersive, moderately dispersive, dispersive and cohesive, moderately cohesive, and cohesive. The questionnaire was not a validated instrument.

\section{Postoperative assessment and analysis}

During the follow-up period of the study, no corneal laser surgeries were permitted for the operative eyes. Follow-up appointments were scheduled for $6 \pm 2$ hours, $24 \pm 4$ hours, $7 \pm 2$ days, $30 \pm 7$ days, and $90 \pm 14$ days. At each of these appointments, IOP and adverse events were recorded. All appointments at 1 day postoperative and later included assessments of corrected distance Snellen visual acuity and of ocular signs by slit-lamp examination. Ocular signs included cells, flare, and corneal edema. Ocular signs were considered clinically significant if $>50$ cells were observed or if flare or edema were rated "severe" (on a scale of none, mild, moderate, or severe). At the final visit, endothelial cell density images were recorded. Examiners were masked to treatment type at all postoperative visits.

Statistical analyses were performed using SAS for Windows (v 9.0; SAS Institute Inc, Cary, NC) and Microsoft Excel (v 2002; Microsoft Corp, Redmond, WA), with statistical significance set at $P<0.05$. Primary efficacy variables were incidence of postoperative IOP $\geq 30 \mathrm{mmHg}$ and changes from baseline in corneal endothelial cell density. Primary safety variables included ocular signs and adverse events. The evaluations of the OVDs by the surgeons were secondary efficacy variables.

\section{Results}

A total of 249 eyes were enrolled in the study, with 128 eyes in the DisCoVisc OVD group and 121 eyes in the Healon OVD group. The two groups were statistically similar in age, gender, race, iris color, preoperative IOP, and baseline endothelial cell density (all $P>0.1$ ), as shown in Table 1 . For all patients, disclosure envelopes were returned to the study 
Table I Preoperative demographics

\begin{tabular}{|c|c|c|}
\hline & $\begin{array}{l}\text { DisCoVisc } \\
\text { OVD group, } \\
n=128\end{array}$ & $\begin{array}{l}\text { Healon } \\
\text { OVD group, } \\
n=|2|\end{array}$ \\
\hline Age, years & $70 \pm 10$ & $69 \pm 9$ \\
\hline \multicolumn{3}{|l|}{ Gender } \\
\hline Men (\%) & $37 \%$ & $37 \%$ \\
\hline Women (\%) & $63 \%$ & $63 \%$ \\
\hline \multicolumn{3}{|l|}{ Race } \\
\hline Caucasian (\%) & $96.7 \%$ & $90 \%$ \\
\hline Black (\%) & $1.6 \%$ & $4 \%$ \\
\hline Asian (\%) & $0 \%$ & $1 \%$ \\
\hline Hispanic (\%) & $1.6 \%$ & $3 \%$ \\
\hline Other (\%) & $0 \%$ & $2 \%$ \\
\hline \multicolumn{3}{|l|}{ Iris color } \\
\hline Brown (\%) & $27 \%$ & $36 \%$ \\
\hline Hazel (\%) & $24 \%$ & $23 \%$ \\
\hline Green (\%) & $7 \%$ & $1.7 \%$ \\
\hline Blue (\%) & $42 \%$ & $38 \%$ \\
\hline Grey (\%) & $0 \%$ & $1.7 \%$ \\
\hline Intraocular pressure $(\mathrm{mmHg})$ & $17.7 \pm 2.0$ & $16.9 \pm 2.2$ \\
\hline Endothelial cell density (cells $/ \mathrm{mm}^{2}$ ) & $2600 \pm 300$ & $2600 \pm 300$ \\
\hline
\end{tabular}

Abbreviation: OVD, ophthalmic viscosurgical device.

sponsor with seals intact, indicating that no unmasking of analysts to treatment type had occurred. All 249 patients had at least one postoperative follow-up visit and all patients were included in the analyses of safety and surgical performance. Missed visits were as follows: one patient in each group at 6 hours postoperative, two patients in the DisCoVisc OVD group at 7 days postoperative, and one patient in each group at 30 days postoperative. Nine patients (four in the Healon OVD group and five in the DisCoVisc OVD group) were excluded from the IOP and endothelial cell density analyses because of protocol violations, including deviations from exclusion/inclusion criteria or use of an off-protocol medication with the surgery.

\section{IOP with time}

Of the 240 patients without protocol violations, an additional 12 patients (eight in the DisCoVisc OVD group and four in the Healon OVD group) were excluded from IOP analysis. Patients were excluded from the IOP analysis if the IOP measurement at $6 \pm 2$ hours postoperative was missing or was more than 30 minutes early (ie, if the measurement intended for 4-8 hours postoperative was recorded at 3 hours and 29 minutes postoperative or earlier). The resultant evaluable population was 115 eyes in the DisCoVisc OVD group and 113 eyes in the Healon OVD group. Mean IOPs with time are shown in Figure 1. Mean IOPs were statistically similar between OVD groups at all time points. All mean IOPs were at least $8 \mathrm{mmHg}$ lower than the prespecified safety limit of $30 \mathrm{mmHg}$.
At 6 hours after surgery, both OVD groups had 15 patients with IOP $\geq 30 \mathrm{mmHg}$, yielding a similar percentage of patients with IOP $\geq 30 \mathrm{mmHg}$ ( $13.0 \%$ of DisCoVisc OVD patients, $13.3 \%$ of Healon OVD patients). At 24 hours after surgery, both OVD groups had seven patients with IOP $\geq 30 \mathrm{mmHg}$ (similar between groups, at $6.1 \%$ of DisCoVisc OVD patients and $6.2 \%$ of Healon OVD patients). By postoperative day 7, only one Healon OVD patient ( $0.9 \%$ of cases) and no DisCoVisc OVD patients had IOP $\geq 30 \mathrm{mmHg}$. No IOPs $\geq 30 \mathrm{mmHg}$ were observed in any patient following postoperative day 13. Overall, similar percentages of patients in the DisCoVisc OVD group (17 of $115,14.8 \%$ of patients) and the Healon OVD group (18 of $113,15.9 \%$ of patients) had an IOP $\geq 30 \mathrm{mmHg}$ at any time during the study. The total numbers of patients with IOP $\geq 30 \mathrm{mmHg}$ that were treated with IOP-reducing therapy at any visit were 12 patients in the DisCoVisc OVD group and 11 patients in the Healon OVD group.

\section{Endothelial cell density}

Of the 240 patients without protocol violations, one patient in the Healon OVD group was excluded from the endothelial cell density analysis because the image of her eye was captured at the peripheral cornea instead of the central corneal. For the remaining evaluable 116 Healon OVD patients and 123 DisCoVisc OVD patients, the percentage loss of endothelial cell density from baseline to postoperative day 90 was lower for eyes in the DisCoVisc OVD group (average $6.4 \%$ loss, with a $95 \%$ confidence interval [CI] range: $\pm 2.3 \%$ loss) than for eyes in the Healon OVD group (average 8.8\% loss, with a $95 \%$ CI range: $\pm 2.3 \%$ ). The percentage loss of endothelial cell density was not statistically different between groups ( $P=0.15$ by analysis of variance). The distribution minimum, median, maximum, and quartiles - of percentage loss of endothelial cell density is shown in Figure 2.

\section{Surgical performance of OVDs}

Viscosity characteristics of the OVDs, as assessed subjectively by the nine surgeons, are shown for three surgical stages in Figure 3. The Healon OVD was rated as cohesive in the majority of cases for each surgical stage: $69 \%$ of cases during capsulorhexis, $85 \%$ of cases during phacoemulsification, and $89 \%$ of cases during OVD removal. In contrast, the DisCoVisc OVD was most frequently rated as "both dispersive and cohesive" during each surgical stage: $45 \%$ of cases during capsulorhexis, $56 \%$ of cases during phacoemulsification, and $51 \%$ of cases during OVD removal. During capsulorhexis and phacoemulsification, the characterizations 


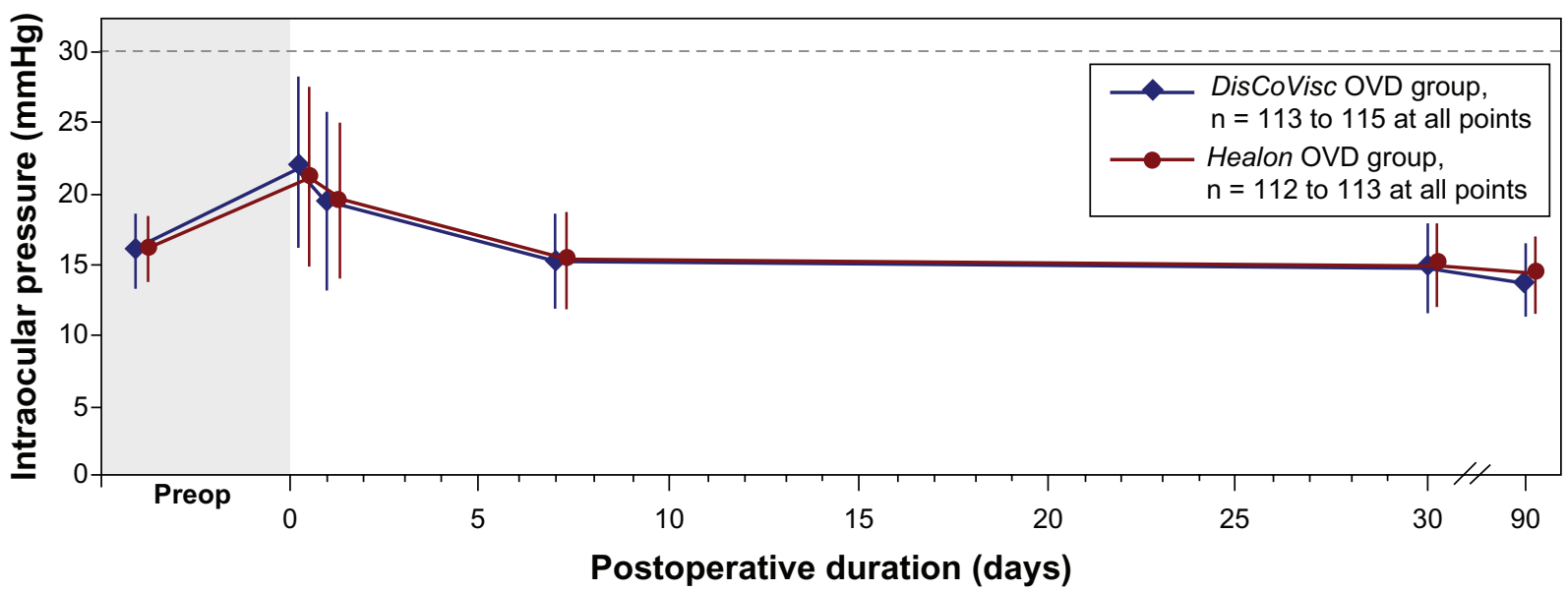

Figure I Mean intraocular pressure. Error bars represent standard deviation. Note break in X-axis. Series are offset in the lateral direction for clarity. Dashed line represents prespecified safety limit.

of DisCoVisc OVD were skewed toward the dispersive side ( $\geq 32 \%$ of cases rated "dispersive" or "moderately dispersive"). During OVD removal, the characterizations of DisCoVisc OVD were skewed toward the cohesive side (37\% of cases rated "cohesive" or "moderately cohesive".) During all three surgical stages, the characterizations of the OVD properties were statistically different between OVD groups $(P<0.0001$ by Cochran-Mantel-Haenszel test).

Space-maintaining characteristics of the OVDs, as assessed subjectively by the nine surgeons, are shown for three surgical stages in Figure 4. When using the DisCoVisc OVD, surgeons most frequently rated the workspace as

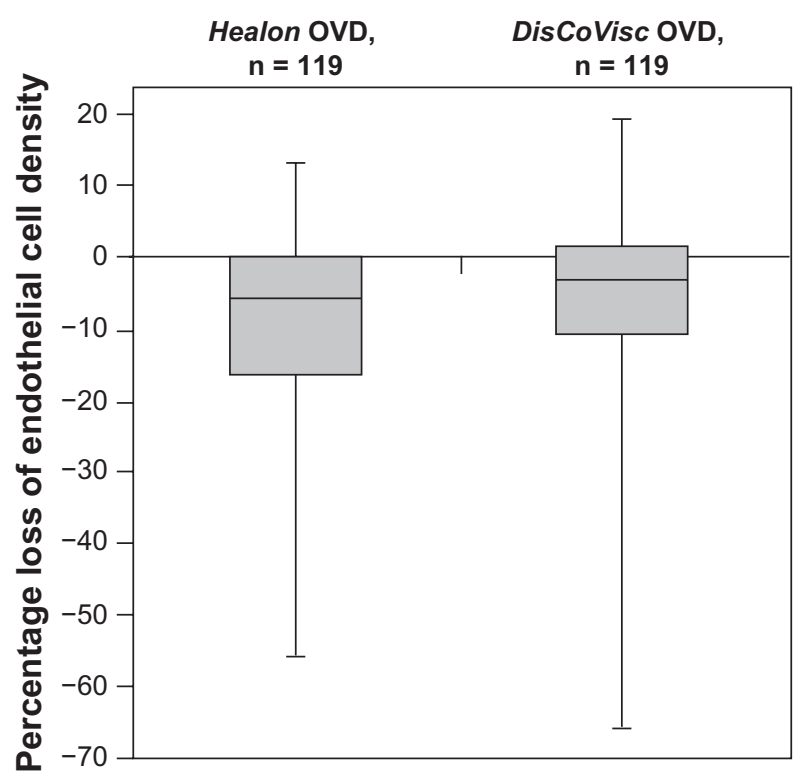

Figure 2 Box plot of percentage change in endothelial cell density from baseline to 90 days postoperative. Bold lines within the boxes represent the median; upper limits of the boxes represent the first quartile; lower limits of the boxes represent the third quartile; and error bars represent the maxima and minima. "full chamber maintained" during each surgical stage: $81 \%$ of cases during capsulorhexis, $78 \%$ of cases during phacoemulsification, and $87 \%$ of cases during IOL insertion. When using the Healon OVD, surgeons most frequently rated workspace as "workspace maintained" during each surgical stage: $37 \%$ of cases during capsulorhexis, $48 \%$ of cases during phacoemulsification, and $49 \%$ of cases during IOL insertion. No cases were rated as having "flat" or "shallow" workspaces during any of the three surgical stages when using the DisCoVisc OVD, but those ratings were given to Healon OVD cases in $32 \%$ of anterior capsulotomies, in $23 \%$ of phacoemulsification stages, and in $3 \%$ of IOL insertions. During all three surgical stages, the characterizations of space maintenance by the OVDs were statistically different between OVD groups $(P<0.0001$ by Cochran-Mantel-Haenszel test).

\section{Safety and adverse events}

No patient in either OVD group exhibited ocular flare that merited a clinically significant score at any of the postoperative examinations. For the aqueous cells parameter, three eyes exhibited clinically significant scores: two eyes in the Healon OVD group (one eye at 1 day postoperative and one eye at 7 days postoperative) and one eye in the DisCoVisc OVD group at an unscheduled visit (41 days postoperative). The latter case was not related to the OVD, but was attributed to residual lens fragments after surgery in an eye with a very small pupil, and was resolved after treatment with prednisolone acetate and atropine. Severe corneal edema was observed in one eye in the DisCoVisc OVD group and in one eye in the Healon OVD group; both cases occurred 1 day postoperative. The most frequently reported clinical observations of events related to the safety and tolerability of 


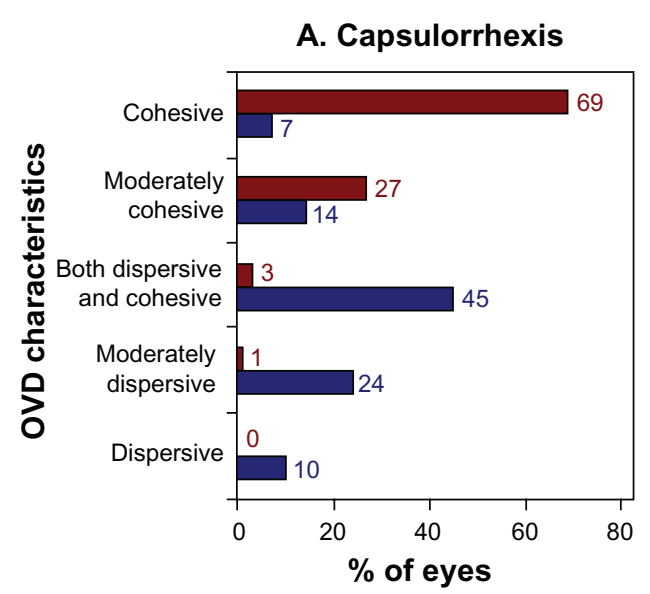

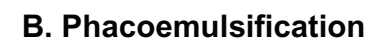

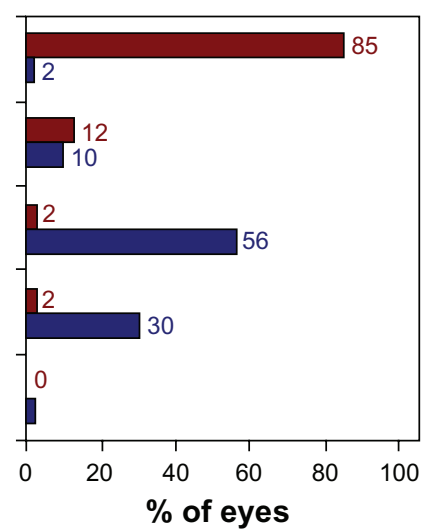

C. OVD removal

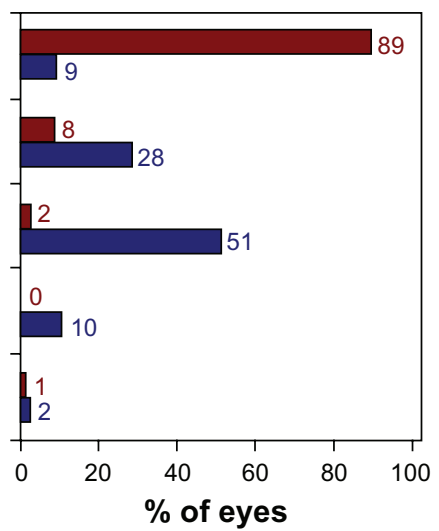

Figure 3 Viscosity characteristics of the ophthalmological viscosurgical devices (OVDs) during three surgical stages. Results are from nine surgeons assessing I2I Healon OVD cases (shown in red) and 128 DisCoVisc OVD cases (shown in blue).

the OVDs occurred in both study groups and included ocular discomfort, conjunctival hyperemia, conjunctival injection, or conjunctival erythema.

\section{Discussion}

In this large, controlled study that did not use prophylactic IOP-lowering medications, the viscous dispersive DisCoVisc OVD group had an IOP profile that was similar to IOP profile of the cohesive Healon OVD comparator group. At 6 hours after surgery, the prevalence of eyes having IOP $\geq 30 \mathrm{mmHg}$ was $13 \%$ in both OVD groups. This prevalence is in accordance with values that were previously reported for eyes that received the cohesive Healon OVD during cataract surgery, ${ }^{13}$ as shown in Table 2. The prevalence is lower than values reported for eyes that received the dispersive Viscoat OVD (Alcon) during cataract surgery $(23 \%-29 \%$ of eyes in
Viscoat OVD groups, Table 2). ${ }^{14-16}$ Judging by IOP profile, DisCoVisc OVD behaved more like a cohesive OVD than like the dispersive OVD Viscoat, even though the chemical compositions of the DisCoVisc and Viscoat OVDs are very similar.

The rheological properties of any OVD arise from the monomer type and polymer formulation of its constituents. In some cases, these constituents provide not only physical protection, but also chemical protection. DisCoVisc viscoelastic contains two biologically relevant glycosaminoglycans: $1.6 \%$ hyaluronic acid (also found in connective tissues) and $4 \%$ chondroitin sulfate (also found in cartilage). ${ }^{1,17}$ Both chondroitin sulfate and hyaluronic acid are antioxidants. ${ }^{18}$ During an in vitro simulation of phacoemulsification, an OVD containing 3\% chondroitin sulfate and 4\% hyaluronic acid (Viscoat OVD) suppressed
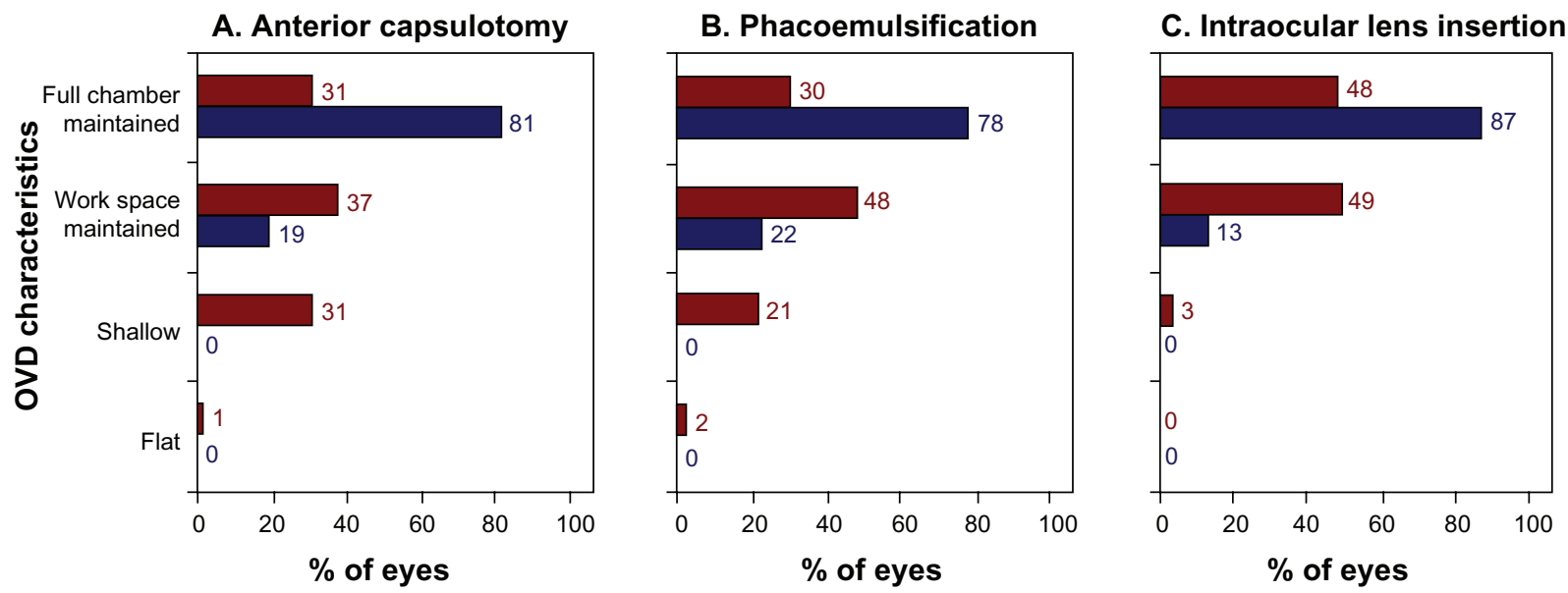

Figure 4 Space-maintaining characteristics of the ophthalmological viscosurgical devices (OVDs) during three surgical stages. Results are from nine surgeons assessing $12 \mathrm{I}$ Healon OVD cases (shown in red) and 128 DisCoVisc OVD cases (shown in blue). 
Table 2 Percentage of eyes with intraocular pressure $\geq 30 \mathrm{mmHg}$ at 6 hours after cataract surgery, as reported in various studies of ophthalmic viscosurgical devices

\begin{tabular}{|c|c|c|c|c|c|}
\hline \multirow[t]{2}{*}{ Reference } & \multicolumn{3}{|c|}{ Ophthalmic viscosurgical devices (OVDs) } & \multirow[t]{2}{*}{ Eyes, $n$} & \multirow{2}{*}{$\begin{array}{l}\text { Eyes } \geq 30 \\
\mathrm{mmHg}, \%\end{array}$} \\
\hline & OVD name & Composition & Character & & \\
\hline \multirow[t]{2}{*}{ This study } & DisCoVisc & $1.6 \% \mathrm{SH}, 4 \% \mathrm{CS}$ & Viscous dispersive & 128 & $13 \%$ \\
\hline & Healon & $\mathrm{I} \% \mathrm{SH}$ & Cohesive & 121 & $13 \%$ \\
\hline \multirow[t]{2}{*}{ Rainer et al ${ }^{16}$} & Viscoat & $4 \% \mathrm{CS}, 3 \% \mathrm{SH}$ & Dispersive & 35 & $29 \%$ \\
\hline & Healon5 & $2.3 \% \mathrm{SH}$ & Viscoadaptive & 35 & $6 \%$ \\
\hline \multirow[t]{2}{*}{ Rainer et al ${ }^{14}$} & Viscoat & $4 \% \mathrm{CS}, 3 \% \mathrm{SH}$ & Dispersive & 40 & $23 \%$ \\
\hline & Ocucoat & $2 \% \mathrm{HPMC}$ & Dispersive & 40 & $5 \%$ \\
\hline Rainer et $\mathrm{a}^{15}$ & Viscoat & $4 \% \mathrm{CS}, 3 \% \mathrm{SH}$ & Dispersive & 38 & $26 \%$ \\
\hline \multirow[t]{2}{*}{ Rainer et $\mathrm{al}^{13}$} & Healon & $\mathrm{I} \% \mathrm{SH}$ & Cohesive & 40 & $13 \%$ \\
\hline & Ocucoat & $2 \% \mathrm{HPMC}$ & Dispersive & 40 & $10 \%$ \\
\hline
\end{tabular}

Abbreviations: CS, chondroitin sulfate; HPMC, hydroxypropyl methylcellulose; SH, sodium hyaluronate.

free radicals significantly more than an OVD containing $2.3 \%$ hyaluronic acid alone (Healon5 OVD, Advanced Medical Optics, Inc). ${ }^{15}$ The relative chemical and rheological protective effects of various OVDs are not yet fully understood.

With regard to the protection of endothelial cells in the current study, the percentage cell loss with the DisCoVisc OVD (average 6.4\% loss) was lower than with the Healon OVD comparator (average 8.8\% loss), though the difference was not statistically significant. It is not clear whether the endothelial protection provided by the OVDs was due to rheological properties, to chemical/antioxidant content, or to a combination of both. Protection could be related to endothelium-coating properties of the OVDs. Two studies using animal eyes found that a thin uniform layer of DisCoVisc OVD remained as a lining on the inner cornea after phacoemulsification and removal of OVD; the investigators suggested that this coating was indicative of the protective effects of the DisCoVisc OVD. ${ }^{19,20}$ The residual layer of DisCoVisc OVD (325 $\mathrm{mm} \pm 164)$ was significantly thicker than the residual layer of Healon OVD $(4 \pm 11 \mu \mathrm{m}) .{ }^{20}$ The coating behavior of an OVD may partially explain its protection of the endothelium.

The values of percentage loss of endothelial cells in the current study (6.4\% loss in the DisCoVisc OVD group and $8.8 \%$ loss in the Healon OVD group) are near the low end of ranges found in similar studies, which reported averages of 4\%-19\% loss. ${ }^{21-23}$ Results with DisCoVisc OVD in the current study are in accord with a literature report of $4 \%$ loss for 50 eyes that received DisCoVisc OVD during cataract surgery. ${ }^{23}$ The DisCoVisc OVD results presented here are also similar to percentage loss reported for eyes that received the viscoadaptive Healon 5 OVD $(6 \% \text { loss })^{22}$ and eyes that received DuoVisc OVD with the soft-shell technique ( $5 \%$ loss $){ }^{23}$

In this study, surgeons subjectively rated DisCoVisc OVD as statistically superior to Healon OVD in maintaining anterior chamber space during anterior capsulotomy, and found that the DisCoVisc OVD demonstrated both cohesive and dispersive properties during capsulorhexis. It may be important that $32 \%$ of surgeons rated the anterior chamber as flat or shallow during capsulorhexis when using Healon OVD. Incision creation and capsulorhexis are important stage-setting steps in determining the ease and success of a cataract surgery. It would be important to distinguish whether surgeons gave the OVDs grades for maintenance of space that depended on their capsulorhexis techniques, either via cystotome only or via cystotome initiation and forceps completion. These surgical techniques were not recorded during this study. Surgeons using cystotomes only for capsulotomy may find that their opinion of chamber space maintenance may be affected by the friction on the needle under the influence of the OVD.

In addition to capsulotomy results, surgeons in this study also subjectively rated DisCoVisc OVD as statistically superior to Healon OVD in maintaining anterior chamber space during phacoemulsification and IOL insertion. Surgeons also found that the DisCoVisc OVD demonstrated both cohesive and dispersive properties during phacoemulsification and removal of OVD. This subjective judgement of the rheological properties of the DisCoVisc OVD is in accordance with findings of a smaller clinical trial $(n=35)^{24}$ and with benchtop experiments that found the DisCoVisc OVD had both cohesive and dispersive qualities, meriting the new classification, "viscous dispersive". ${ }^{2}$ During OVD removal in this study, the characterizations of DisCoVisc OVD were 
skewed toward the cohesive side. This result is in accordance with the results of an animal study in which the speed of DisCoVisc OVD removal was second only to the removal of the cohesive Provisc OVD (Alcon Laboratories, Inc) in an array of four different OVDs. ${ }^{25}$ Similarly, another animal study found that fluorescein-stained DisCoVisc OVD stayed in the eye during phacoemulsification and then was removed in a single mass at the end of the procedure. ${ }^{26}$

One uncontrolled variable in this study was surgical technique. Some researchers have reported that endothelial cell loss was less with the phaco-chop technique than with the divide-and-conquer technique, ${ }^{27,28}$ though other researchers have found that the two methods produced similar endothelial outcomes. ${ }^{29}$ It would be interesting to know whether patients treated by one particular surgeon in this study received different benefits due to surgical technique, but surgical techniques were not recorded in this study. Moreover, identifying beneficial surgical strategies would be helpful in establishing practices to use the OVDs to greatest advantage.

\section{Conclusion}

This study found that the DisCoVisc OVD had rheological characteristics that made it suitable for the entire surgical procedure, and that DisCoVisc OVD was safe, well-tolerated, and protective in eyes undergoing cataract extraction and IOL implantation.

\section{Acknowledgments}

This study was funded and analyzed by Alcon Research, Ltd; they also assisted with preparation of this manuscript.

\section{Disclosure}

Dr Modi is a member of Alcon Speakers Alliance. Dr Davison is a consultant to Alcon.

\section{References}

1. Pandey S, Thakur J, Werner L, Izak A, Apple D. Update on ophthalmic viscosurgical devices. In: Agarwal S, Agarwal A, Agarwal A, editors. Phacoemulsification. 3rd ed. Boca Raton, FL: Taylor and Francis. 2004:179-195.

2. Arshinoff SA, Jafari M. New classification of ophthalmic viscosurgical devices-2005. J Cataract Refract Surg. 2005;31(11):2167-2171.

3. Bollinger K, Smith S. Ophthalmic viscosurgical devices. In: Henderson B, editor. Essentials of Cataract Surgery. Thorofare, NJ: Slack Inc. 2007: 63-68.

4. Arshinoff SA. Dispersive-cohesive viscoelastic soft shell technique. J Cataract Refract Surg. 1999;25(2):167-173.

5. Bourne WM, McLaren JW. Clinical responses of the corneal endothelium. Exp Eye Res. 2004;78(3):561-572.

6. Yen PC, Colby K. Corneal endothelial dystrophies. In: Foster CS, Azar DT, Dohlman CH, editors. Smolin and Thoft's The Cornea. 4th ed. Philadelphia, PA: Lippincott Williams \& Wilkins. 2004:845-874.
7. Mastropasqua L, Carpineto P, Ciancaglini M, Falconio G. Intraocular pressure changes after phacoemulsification and foldable silicone lens implantation using Healon GV. Ophthalmologica. 1998;212(5): 318-321.

8. Thirumalai B, Blamires TL, Brooker L, Deeks J. Heavier molecular weight ocular viscoelastic devices and timing of post-operative review following cataract surgery. BMC Ophthalmol. 2007;7:2.

9. Vajpayee R, Verma K, Sinha R, Titiyal J, Pandey RM, Sharma N. Comparative evaluation of efficacy and safety of ophthalmic viscosurgical devices in phacoemulsification [ISRCTN34957881]. BMC Ophthalmol. 2005;5(1):17.

10. Tan JH, Newman DK, Klunker C, Watts SE, Burton RL. Phacoemulsification cataract surgery: is routine review necessary on the first post-operative day? Eye. 2000;14(Pt 1):53-55.

11. Thirumalai B, Baranyovits PR. Intraocular pressure changes and the implications on patient review after phacoemulsification. $J$ Cataract Refract Surg. 2003;29(3):504-507.

12. International Organization for Standardization. Ophthalmic Implants Ophthalmic Viscosurgical Devices. ISO 15798 ed. 2001.

13. Rainer G, Schmid KE, Findl O, et al. Natural course of intraocular pressure after cataract surgery with sodium hyaluronate $1 \%$ versus hydroxypropylmethylcellulose 2\%. Ophthalmology. 2007;114(6):1089-1093.

14. Rainer G, Menapace R, Findl O, et al. Intraocular pressure rise after small incision cataract surgery: a randomised intraindividual comparison of two dispersive viscoelastic agents. Br J Ophthalmol. 2001;85(2): 139-142.

15. Rainer G, Menapace R, Schmid KE, et al. Natural course of intraocular pressure after cataract surgery with sodium chondroitin sulfate 4\%-sodium hyaluronate 3\% (Viscoat). Ophthalmology. 2005;112(10): 1714-1718.

16. Rainer G, Menapace R, Findl O, Georgopoulos M, Kiss B, Petternel V. Intraocular pressure after small incision cataract surgery with Healon5 and Viscoat. J Cataract Refract Surg. 2000;26(2):271-276.

17. DisCoVisc Ophthalmic Viscosurgical Device [package insert]. Fort Worth, TX: Alcon Laboratories, Inc. 2007.

18. Campo GM, Avenoso A, Campo S, Ferlazzo AM, Calatroni A. Antioxidant activity of chondroitin sulfate. In: Volpi N, editor. Adv Pharmacol. 2006:418-431.

19. Yoshino M, Bissen-Miyajima H, Ohki S. Residual amounts of ophthalmic viscosurgical devices on the corneal endothelium following phacoemulsification. Jpn J Ophthalmol. 2009;53(1):62-64.

20. Petroll WM, Jafari M, Lane SS, Jester JV, Cavanagh HD. Quantitative assessment of ophthalmic viscosurgical device retention using in vivo confocal microscopy. J Cataract Refract Surg. 2005;31(12): 2363-2368.

21. Storr-Paulsen A, Norregaard JC, Farik G, Tarnhoj J. The influence of viscoelastic substances on the corneal endothelial cell population during cataract surgery: a prospective study of cohesive and dispersive viscoelastics. Acta Ophthalmol Scand. 2007;85(2):183-187.

22. Holzer MP, Tetz MR, Auffarth GU, Welt R, Völcker HE. Effect of Healon 5 and 4 other viscoelastic substances on intraocular pressure and endothelium after cataract surgery. J Cataract Refract Surg. 2001;27(2): 213-218.

23. Praveen MR, Koul A, Vasavada AR, Pandita D, Dixit NV, Dahodwala FF. DisCoVisc versus the soft-shell technique using Viscoat and Provisc in phacoemulsification: randomized clinical trial. $J$ Cataract Refract Surg. 2008;34(7):1145-1151.

24. Gibelalde A, Mendicute J, Bidaguren A, Irigoyen C. Prospective randomized trial comparing Discovisc versus Healon in phacoemulsification. Arch Soc Esp Oftalmol. 2007;82(8):489-494. Spanish.

25. Oshika T, Okamoto F, Kaji Y, et al. Retention and removal of a new viscous dispersive ophthalmic viscosurgical device during cataract surgery in animal eyes. Br J Ophthalmol. 2006;90(4):485-487.

26. Bissen-Miyajima H. In vitro behavior of ophthalmic viscosurgical devices during phacoemulsification. J Cataract Refract Surg. 2006;32(6):1026-1031. 
27. Pirazzoli G, D'Eliseo D, Ziosi M, Acciarri R. Effects of phacoemulsification time on the corneal endothelium using phacofracture and phaco chop techniques. J Cataract Refract Surg. 1996;22(7):967-969.

28. Zetterstrom C, Laurell CG. Comparison of endothelial cell loss and phacoemulsification energy during endocapsular phacoemulsification surgery. J Cataract Refract Surg. 1995;21(1):55-58.
29. Storr-Paulsen A, Norregaard JC, Ahmed S, Storr-Paulsen T, Pedersen TH. Endothelial cell damage after cataract surgery: divide-and-conquer versus phaco-chop technique. J Cataract Refract Surg. 2008;34(6): 996-1000.

\section{Publish your work in this journal}

Clinical Ophthalmology is an international, peer-reviewed journal covering all subspecialties within ophthalmology. Key topics include: Optometry; Visual science; Pharmacology and drug therapy in eye diseases; Basic Sciences; Primary and Secondary eye care; Patien Safety and Quality of Care Improvements. This journal is indexed on

Submit your manuscript here: http://www.dovepress.com/clinical-ophthalmology-journal

\section{Dovepress}

PubMed Central and CAS, and is the official journal of The Society of Clinical Ophthalmology (SCO). The manuscript management system is completely online and includes a very quick and fair peer-review system, which is all easy to use. Visit http://www.dovepress.com/ testimonials.php to read real quotes from published authors. 